\title{
Humanismo tecnológico: fundamento para una inteligencia artificial responsable
}

\author{
Technological humanism: basis for responsible artificial intelligence
}

\author{
Antonio Luis Terrones ${ }^{1}$
}

Fecha de recepción: 4-6-2019

Fecha de aprobación: 30-9-2019

\begin{abstract}
Resumen
Los rápidos avances en el campo de la inteligencia artificial (IA) en las últimas décadas ponen de relieve la necesidad de incorporar criterios éticos de responsabilidad. Esa incorporación permitirá el planteamiento de una inteligencia artificial responsable (IAR) que tendrá que fundamentarse en la formulación de un nuevo humanismo. Se entiende que a partir de un humanismo tecnológico se podría impulsar la IAR en el contexto tecnológico actual. Los desafíos de la IA imponen el imperativo de plantear un humanismo de este tipo que asuma un compromiso con miras al futuro. En ese sentido, el humanismo tecnológico representa la exigencia de un tiempo de desafíos tecnológicos y a la vez una premisa ineludible en el planteamiento de la IAR. Es una premisa para la responsabilidad ante un tiempo de exigencias que no se pueden esquivar. Los importantes y profundos avances que ha experimentado el campo de la tecnología nos sitúan frente a un escenario novedoso para la humanidad que demanda una nueva contextualización del humanismo. A la vez, este humanismo tecnológico es crítico porque es conocedor del límite y de su condición condicionada, es decir, de su posición de carestía que la invita a imaginar lo que puede ser y hasta dónde puede llegar. Esta invitación permite entender que los límites no se encuentran situados en un plano negativo, sino más bien como un avistamiento del lado positivo de la tecnología como espacio desde el que hacer posible el florecimiento humano y un nuevo modo de obrar humano. Palabras clave: Tecnología, inteligencia artificial, ética, responsabilidad, humanismo tecnológico.
\end{abstract}

\begin{abstract}
The rapid advances in the field of artificial intelligence (AI) in recent decades highlight the need to incorporate ethical criteria of responsibility. This incorporation will allow the approach of a responsible artificial intelligence (IAR) that will have to be based on the formulation of a new humanism. It is understood that from a technological humanism the IAR could be promoted in the current technological context. The challenges of the AI impose the imperative to pose a humanism of this type that assumes a commitment with a view to the future. In that sense, technological humanism represents the demand for a time of technological challenges and at the same time an inescapable premise in the IAR's approach. It is a premise for responsibility before a time of demands that can not be avoided. The important and profound advances that the field of technology has experienced place us in front of a novel scenario for humanity that demands a new contextualization of humanism. At the same time, this technological humanism is critical because it is aware of the limit and its conditioned condition, that is, of its position of scarcity that invites it to imagine what it can be and how far it can go. This invitation allows us to understand that the limits are not located in a negative plane, but rather as a sighting of the positive side of technology as a space from which to make possible the human flowering and a new way of working human.
\end{abstract}

Keywords: Technology, artificial intelligence, ethics, responsibility, technological humanism.

\footnotetext{
1 Docente e investigador de la Escuela de Filosofía de la Pontificia Universidad Católica de Ecuador. Ecuador Correo electrónico: antonioluis.terrones@gmail.com
} 


\section{Introducción}

Las tecnologías más avanzadas, entre las que se encuentra la inteligencia artificial (IA), han experimentando importantes avances que comprometen la vida del ser humano en sus diversas dimensiones. En ese sentido, la pregunta por el ser humano sugiere la necesidad de contextualizar el humanismo en este nuevo escenario tecnológico. En primer lugar, el humanismo representa un impulso frente a determinados imperativos de un tiempo que en ocasiones se formulan en forma de respuesta a los desafíos que acontecen. Por ello, el humanismo tecnológico supone el impulso del tiempo tecnológico que se vive en la actualidad. Un impulso en forma de respuesta que debe formularse de manera integral, cuestionando el discurso de las dos culturas enfrentadas y promoviendo un enriquecimiento de perspectivas epistemológicas para enfrentar las complejas problemáticas de carácter plural.

El humanismo también presenta un carácter proyectivo espacio temporal que responde a las necesidades circunstanciales e imaginarias del ser humano. Pero este carácter va acompañado del conocimiento de los propios límites de la tecnología, es decir, de la asunción de conciencia sobre el condicionamiento limitante que existe sobre nuestros posibles y su impacto en el mundo en función de criterios éticos de responsabilidad.

Durante el siglo XIX existió una falsa oposición entre la técnica y la cultura que no llevó a ninguna parte. Así pues, es importante dejar claro que el humanismo tecnológico no es una invitación para la oposición a la técnica, sino para pensarla en sus amplias dimensiones. A colación de lo dicho hasta ahora, es importante rescatar unas palabras de Simondon:

Este estudio está animado por la intención de suscitar una toma de conciencia del sentido de los objetos técnicos. La cultura se ha constituido en sistema de defensa contra las técnicas; ahora bien, esta defensa se presenta como una defensa del hombre, suponiendo que los objetos técnicos no contienen realidad humana [...] La toma de conciencia de los modos de existencia de los objetos técnicos debe ser efectuada por el pensamiento filosófico, que se encuentra en la posición de tener que cumplir en esta obra un deber análogo al que cumplió en la abolición de la esclavitud y la afirmación del valor de la persona humana. La oposición que se ha erigido entre la cultura y la técnica, entre el hombre y la máquina, es falsa y sin fundamentos; sólo recubre ignorancia o resentimiento. Enmascara detrás de un humanismo fácil una realidad rica en esfuerzos humanos y en fuerzas naturales, y que constituye el mundo de los objetos técnicos, mediados entre la naturaleza y el hombre [...] La mayor causa de alienación en el mundo contemporáneo reside en este desconocimiento de la máquina, que no es una alienación causada por la máquina, sino por el no-conocimiento de su naturaleza y de su esencia, por su ausencia de mundo de significaciones, y por su omisión en la tabla de valores y de conceptos que forman parte de la cultura (Simondon, 2008, pp. 31-32).

No será sugerido bajo ningún concepto lo que Simondon define como un "humanismo fácil", a saber, esa oposición inútil entre la cultura y la técnica, en la que el humanismo ve a la máquina como a un enemigo, ni tampoco en la que el campo ingenieril ve al hombre como ausente en la incidencia del desarrollo maquinal, según lo que plantea el determinismo de Jacques Ellul (2003, 2004). Por lo tanto, ni romanticismo humanista, ni tecnocratismo determinista, sino que se promoverá la formulación de un nuevo relato que supere las absurdas enemistades entre el hombre y la máquina, un humanismo tecnológico. El objetivo que es perseguido en este artículo es el de proponer una fundamentación filosófica desde la que el humanismo tecnológico pueda ser elaborado con base en premisas teóricas que, son consideradas muy relevantes para abordar el fenómeno de la tecnología y para la elaboración de estrategias que caminen hacia el planteamiento de una inteligencia artificial responsable (IAR). y la búsqueda de un nuevo modo de obrar humano. 


\section{El humanismo tecnológico como impulso de un tiempo}

Retomando la aclaración que se hacía al comienzo de este capítulo, el humanismo tecnológico no asume las tesis de aquellos discursos que versan sobre la deshumanización de la técnica y que apelan a la supuesta existencia de una esencia humana inmutable, como en ocasiones refiere Hans Jonas (1995, 1997, 2001, 2005). Es difícil hablar de una esencia humana que permanece en el espacio y en el tiempo, pues el ser del humano es de un espacio y de un tiempo y se construye con base en esas coordenadas. El discurso de la deshumanización de la técnica suele implicar una demonización de esta y, en ocasiones, pretende esquivar ciertas responsabilidades. El impulso para formular un humanismo tecnológico nace, no de una mirada sobre la técnica vista como amenaza, ni tampoco de la pretensión de su humanización, sino de un reconocimiento de la misma como un universo de posibilidades que es enfrentada desde los límites humanos.

El humanismo tecnológico parte de un escenario en el que la teoría de las dos culturas es reflexionada. Charles Percy Snow (2013) sugiere, de forma optimista, la necesidad de construir un vínculo entre científicos y literatos en beneficio de la humanidad. La propuesta de encuentro cultural de Snow radica en la urgencia de transformar la educación de su tiempo para adecuarla a las nuevas posibilidades y retos que plantea la revolución científica ${ }^{2}$. Ante aquella tradición de pensamiento que afirma la existencia de dos culturas, separando de forma errónea y tajante las ciencias de las humanidades, como si de dos dimensiones del conocimiento humano se trataran, hay que señalar que no existen dos culturas diferenciadas, como si una perteneciera al conocimiento científico y otra al conocimiento humanista vinculado con aquello que denominan "tradición" (Huxley, 2017). Según esta forma de pensar, las humanidades mirarían al pasado y serían las guardianas de la tradición, en cambio, las ciencias serían las encargadas de mirar al futuro y de asumir la tarea del progreso. Concebir las ciencias y las humanidades como compartimentos separados, ajenos y cerrados, representa un serio obstáculo para el humanismo tecnológico. Esto supone un error porque el humanismo reconoce al ser humano en todas sus dimensiones sin dejar de lado ninguna, ya que el conocimiento es un elemento esencial del ser humano y no puede considerarse de forma compartimentada, sino integral. No podemos asumir la responsabilidad de formular un humanismo tecnológico si antes no superamos el discurso errado de las dos culturas.

El ser humano no puede prescindir de ninguna de sus dimensiones para fortalecer su condición, pues todas las partes se encuentran imbricadas, como si de unos engranajes se trataran. El ser humano es un ser integral, sus partes están interrelacionadas, y sus condiciones son variadas. Es importante reconocer este tema de las dimensiones imbricadas para no caer en la simplicidad de desestimar ciertos saberes que también forman parte de nuestro conocimiento y de nuestra existencia. Tampoco debemos esquivar la idea de que el ser humano desarrolla un ejercicio hermenéutico para poder entender la realidad a la que se enfrenta, y es precisamente en ese ejercicio donde surge un importante vínculo entre los saberes que el humano ha formulado para dotarse de una condición de integridad.

Los importantes y profundos avances, que ha experimentado el campo de la tecnología, nos sitúan frente un escenario novedoso para la humanidad, que requiere de una contextualización del humanismo. Anteriormente se ha mencionado que el ser del humano pertenece a un tiempo y un espacio concretos y que esa es la razón por la que existe la necesidad de que el humanismo de nuestro tiempo se reformule con base en unas exigencias diferentes respecto a las de otras épocas históricas. Este proyecto humanista no se entiende como una recreación de los humanismos del pasado, sino que asume la responsabilidad de un nuevo tiempo, pues es un compromiso que se asume ante el fenómeno tecnológico. Representa un nuevo espíritu que es impulsado en medio

2 Es importante señalar que John Brockman (1996) se aleja de lo que propuso Snow, pues la conciliación de las dos culturas no puede circunscribirse exclusivamente al ejercicio comunicativo y divulgativo de los científicos para el gran público, pues en realidad, hay aspectos propios de las humanidades sobre los cuales la tradición científica debería reflexionar para enriquecer su actividad, otorgándoles un espacio que evite la colonización cognoscitiva y cultivando un carácter más plural en términos epistemológicos y metodológicos. 
de la oleada tecnológica. En ese sentido, es pertinente considerar este humanismo como un compromiso de responsabilidad ante los desafíos tecnológicos que nos depara el campo de la IA y que exigen un nuevo impulso.

El humanismo siempre ha surgido de impulsos motivados por los contextos espacio temporales concretos. Puede recordarse el humanismo renacentista, caracterizado por una apertura grandiosa del conocimiento con la explosión de las artes y las ciencias. Este impulso humanista nace del deseo de buscar respuestas y necesita de una salida de lo cotidiano, de esas situaciones que nos abocan a un sonambulismo, que en este tiempo sería de carácter tecnológico (Winner, 2008). Es un impulso hacia el esclarecimiento, por la búsqueda de posibilidades que permitan un florecimiento con miras al futuro, pero sin olvidar el legado histórico como reconocimiento. El pasado no puede representar un obstáculo desde el que reformular el presente y construir el futuro a partir de una mirada posibilitadora que arroje luz al tiempo tecnológico en provecho del ser humano.

Este impulso debe ir acompañado de una superación del discurso de las dos culturas, ya que dificulta una formación integral del ser humano y promueve seres humanos fragmentarios (Aguirre Romero, 2002, p. 9). Este humanismo se aleja de su razón de ser cuando renuncia a sus pretensiones de integridad y a la totalidad como horizonte, que es precisamente donde la tecnología tiene mucho que aportar en favor del proyecto de un nuevo humanismo.

\section{La escritura de un relato de proyección}

Recordando las reflexiones ofrecidas en la Meditación de la técnica de José Ortega y Gasset (1965), podría afirmarse que somos seres con un gran poder imaginario, pues más allá de la realidad presente, imaginamos un abanico de posibilidades. José Luis Molinuevo acierta cuando, recuperando la alegoría de la caverna de Platón, afirma que "somos productores y consumidores de imágenes" (2004, p. 170). Esa afirmación significa que vivimos en la frontera entre dos mundos que se funden gracias a la posibilidad, el mundo real y el mundo de la imaginación. Pero no porque nos situemos frente a un mundo de imaginación deberíamos adoptar una posición negativa, quietista, descalificadora, que es la que muchas veces ciertos postulados pesimistas ofrecen, como el de Nicholas Carr, quien titula una de sus obras más importantes como Atrapados: como las máquinas se apoderan de nuestras vidas.

El humanismo tecnológico es un humanismo limitado, porque así debe ser si lo consideramos como premisa fundamental de una IAR, ¿pero qué quiere decir limitado? Limitado en el sentido que no orienta su actividad tecnológica hacia cualquier cosa que imagine, evitando de ese modo cualquier objeción ética. Es un humanismo limitado porque es más intensivo que extensivo, en palabras de Molinuevo (2004, p. 170), ya que el interés está orientado hacia su henchimiento y no hacia su capricho ilimitado, sino que más bien es finito, conocedor de sus límites y comprometido con su responsabilidad consciente. Aquello a lo que llamamos "humano" puede ser reescrito dentro de unos límites tecnológicos que asuman los desafíos con conciencia y compromiso, para que aquel relato que sea pretendido impulsar incorpore premisas éticas como la responsabilidad. En ese sentido, algo de razón llevaba Sartre (1999) cuando sostenía que somos libres para hacernos y que eso implica una responsabilidad de la que no podemos despojarnos. También deberíamos recordar a Ortega y Gasset, pues no olvidemos que el filósofo español insiste a lo largo de su obra en que el hombre se hace a partir de sus circunstancias, y con él podríamos afirmar que las circunstancias nos empujan a ser conscientes de cuáles son nuestros límites y a orientar nuestra acción en función de los mismos. Esta es una visión realista del ser humano y representa un proyecto diferente al que infunde la visión humanista propia de un pensamiento renacentista como el de Pico della Mirandola (1984). La visión realista se sitúa como antecedente en la fundación del humanismo tecnológico, ya que reconoce los límites de nuestra condición y ciertas distopías en la acción tecnológica, lo que dado el caso de estas páginas invitaría a pensar sobre la necesidad de asumir responsabilidad.

El humanismo tecnológico no es un humanismo idealista, pues no parte de la totalidad de la humanidad como premisa fundacional, sino del reconocimiento de un 
individuo. El individuo se sitúa en una posición relacional, alejándose de la visión de sustancia o subsistencia, propia del cartesianismo (Molinuevo, 2004, p. 178). El ser humano es un ser necesitado, por eso el humanismo es del individuo, porque es un humanismo que reconoce una necesidad relacional e identifica en la tecnología una oportunidad de enriquecimiento existencial. Frente a esta postura de un humanismo relacional y reconocedor de sus necesidades, se encuentra un humanismo anticuado, de otro tiempo, que es el causante de los grandes males que la humanidad ha sufrido, precisamente por no reconocer al ser humano como un ser necesitado, sino más bien como un ser auténtico y esencialista.

Anteriormente han sido mencionados los límites como una de las fronteras características del ser humano. Pues bien, es precisamente en la observación de esos límites donde se encuentra el reconocimiento de la necesidad y de la búsqueda de relaciones. Molinuevo también habla de un humanismo del límite, siguiendo a Eugenio Trías (2000). Esta condición limítrofe allana el camino para hablar de un humanismo tecnológico. El distanciamiento del humanismo esencialista e idealista permite abrir la puerta a una perspectiva que reconoce nuestras limitaciones, lo que sirve como impulso para una mirada orientada al futuro, que es donde entra en escena la tecnología. No obstante, el conocimiento de los límites no sirve como soporte para proyectos que asuman la necesidad de llegar a los límites de las posibilidades, sino más bien para aprovechar las posibilidades que nuestra condición de seres limitados nos brinda. Es en ese punto donde reside el momento en el que el ser humano se convierte en ser técnico y comienza a ver la posibilidad de una sobrenaturaleza, como diría Ortega (1965). Además, pensemos que la tecnología nos invita a repensar la realidad y a observar nuevas posibilidades de realización de nuestra existencia, ya que estamos inmersos en un mundo inevitablemente tecnológico, y lo importante no es situarse con una actitud que desestime, sino que más bien aproveche las oportunidades que se nos brindan para nuestro florecimiento.

Por lo tanto, el humanismo tecnológico es un humanismo reconocedor del límite y a la vez de su condición "condicionada”, es decir, de su carácter de necesidad que le invita a imaginar lo que puede ser. Esta invitación permite entender que los límites no se encuentran situados en un plano negativo, que incluso podría considerar a la técnica como causante de sus males y pérdida de una supuesta esencia, sino más bien como un avistamiento del lado positivo de la técnica como ese espacio desde el que hacer posible el florecimiento.

Junto con José Ortega y Gasset y Jean Paul Sartre se puede afirmar que la vida está por hacerse y que la experiencia vital encuentra su razón de ser en la dimensión proyectiva, en un sentimiento estético de la misma donde somos responsables de escribir nuestro relato. La conciencia del límite nos empuja a poder ser, a mantenernos a flote frente a las circunstancias de la vida. La tecnología pone de relieve nuestras limitaciones, nos hace ser conscientes de estas y nos brinda la posibilidad de imaginar, de poder ser. Ahí es donde radica la necesidad de un humanismo tecnológico, en esa visión optimista de la tecnología, pero no acrítica, donde los inventos tecnológicos nos presentan un universo de posibilidades apremiantes para nuestra existencia. Frente a este optimismo crítico tecnológico también hay que examinar la existencia de inventos que no favorecen el florecimiento humano y a los que hay que anteponer un humanismo tecnológico, no con las pretensiones de humanizar la técnica, o de que las humanidades fagociten el mundo tecnológico, sino más bien como reorientación de las intencionalidades originarias.

En ese sentido, está reorientación de la acción tecnológica en función del nuevo humanismo no gira en torno a la idea de la formulación de un humano de corte idealista que domine el mundo, sino de un ser respetuoso con la realidad, responsable y consciente de sus límites y posibilidades en función de las circunstancias, en definitiva, que vea en la tecnología el reconocimiento de su ser marginal para proyectarse en ella y sacar el máximo partido para su beneficio existencial con prudencia. Como señala Molinuevo: "no se trata de ir con las tecnologías al límite de las posibilidades humanas, sino de extraer las posibilidades del límite humano" (2004, p. 190). 


\section{Humanismo tecnológico y pragmatismo}

El humanismo tecnológico que está propuesto tiene una raíz pragmática, donde el conocimiento teje un hilo conductor con la experiencia en la búsqueda de la resolución de problemáticas. Este humanismo se fundamenta, en cierto modo, en la experiencia humana donde la técnica representa un elemento constitutivo, según John Dewey (1948). Cuando ha sido señalado en este apartado que lo que se pretende con el humanismo tecnológico es reorientar la tecnología en beneficio del florecimiento humano, ha sido consciente de la posibilidad de llevar a cabo ese proyecto. Pues podría entenderse que es posible debido a que nuestra condición técnica como seres humanos está estrictamente dirigida hacia la resolución de problemáticas y por lo tanto existen motivos para pensar que tal reorientación es viable. Es importante una fundamentación pragmática de la técnica, que Dewey describe de la siguiente manera:

'Tecnología' significa todas las técnicas inteligentes por las que las energías de la naturaleza y del hombre son dirigidas y utilizadas en la satisfacción de las necesidades humanas, no se puede limitar a unas pocas, exteriores y relativamente mecánicas formas. A la vista de sus posibilidades, la concepción tradicional de experiencias es obsoleta (Dewey, 1984, p. 277).

La tecnología al servicio del florecimiento humano, esa es la dimensión pragmática del humanismo tecnológico en términos existenciales. Nuestra condición técnica organizaría la acción tecnológica generando mecanismos que beneficien el camino para el provecho humano. Además, debido a la evidente a la utilidad, las acciones tecnológicas tendrían que incorporar criterios de responsabilidad para no desviarse del pensamiento útil. No obstante, cuando se menciona la utilidad no se está pensando en ningún momento en una utilidad propia de la esfera de la racionalidad instrumental que tanto criticaban Max Horkeimer y Theodor Adorno (2010, 2016). Las herramientas tecnológicas pueden impulsarnos hacia la realización de aquellos proyectos que consideremos beneficiosos para nuestra existencia en el mundo y para vivir mejor.
Otra premisa ineludible en el proyecto del humanismo tecnológico tiene que ver con el poder de la inteligencia colectiva, pues es importante considerar que es un tema muy relevante y desafiante para la educación de nuestro tiempo. El Massachusetts Institute of Technology (MIT) fundó, en 2006, el Center for Collective Intelligence, una entidad que tenía la finalidad de reunir a diferentes investigadores para trabajar de forma conjunta y poder esclarecer cómo la tecnología está cambiando nuestra forma de trabajar juntos, pues entendió que la inteligencia colectiva abre un abanico de posibilidades a organizaciones e instituciones para pensar de manera diferente (Parra y Arenas-Dolz, 2015, p. 115). La humanidad ha trabajado de forma conjunta desde sus inicios y lo que supone un reto en la actualidad es el poder articular procesos para que los sistemas de IA puedan servir para fortalecer la interconexión intelectual y de ese modo fortalecer la formación de conocimiento en los espacios educativos. Como señalan Parra y Arenas-Dolz (2015):

La meta de la inteligencia colectiva es el reconocimiento mutuo y el enriquecimiento de las personas. Para ello es necesario que esas personas puedan conversar e interactuar, lo que resulta muy sencillo hoy en día gracias a la tecnología. Internet favorece el intercambio de ideas y conocimientos. Con la Web 2.o aparecen nuevas formas de relacionarse, en las que los consumidores pasan a ser también creadores, como consecuencia de la facilidad para portar información [...] El conocimiento absoluto no es posible. Es por esta razón que resulta casi vital la colaboración para «el reconocimiento y el enriquecimiento mutuo de las personas, y no el culto de comunidades fetichizadas o hipostasiadas». El intercambio de conocimiento y experiencias, donde la diferencia es una manera de enriquecer el saber, nos aleja de una uniformada de pensamiento [...] De esta manera, si juntamos todos esos microsaberes, crearemos una inteligencia colectiva, que parte del principio de que cada persona sabe sobre algo. Por tanto nadie tiene el conocimiento absoluto. De lo que resulta fundamental la inclusión y participación de los conocimientos de todos (p. 123). 
La tecnología incide en los procesos cognitivos del ser humano y el diseño de redes de formación de conocimiento colectivo por medio de intelectos sintéticos podría servir para forjar nuevos hábitos educativos que fortalecerían el nacimiento de nuevas ideas que dieran respuestas a los nuevos desafíos tecnológicos. La suma de perspectivas permite articular la búsqueda de soluciones y alternativas de mejor manera y en ese aspecto la IA podría tender puentes, lo que supondría un beneficio para la humanidad. La clave está en buscar mecanismos que sirvan de bisagra de unión entre los seres humanos, corrigiendo aquellos productos tecnológicos que dificultan la construcción de redes de conocimiento y que tienden al aislamiento de los sujetos. La IA puede estar al servicio de la formación de inteligencia colectiva para el provecho de la humanidad. Este es precisamente un punto fuerte para la formación educativa de las próximas décadas, una IAR que acompañe los procesos de aumento de la inteligencia colectiva, creandose esa forma una nueva cultura de conocimiento, en vez de ahondar más en el problema del individualismo y el aislamiento humano, que es a lo que están conduciendo algunas tecnologías como el teléfono móvil, del que muchas personas hacen un mal uso. Además, la inteligencia colectiva promueve aquellas ideas que sirven para combatir conocimientos que se consideran absolutos y uniformes, lo que supone, en muchos casos, un obstáculo para la democracia y la justicia.

El humanismo tecnológico también se encuentra en una posición de servicio a las humanidades, esos otros saberes que están siendo olvidados y desplazados durante los últimos tiempos y que pueden ser fortalecidos por medio de los nuevos medios tecnológicos. Este humanismo se escribe sobre un nuevo relato en el que se incorporan perspectivas filosóficas que pueden enriquecer, ampliar la racionalidad y pensar la condición humana. Una pérdida de la hegemonía de lo estrictamente positivo e instrumental requiere de la búsqueda de un fundamento en otras propuestas filosóficas dque ofrecen importantes aprendizajes. Una de las claves para el humanismo tecnológico se encuentra en la superación del tipo de racionalidad sobre la que se ha configurado el mundo.

\section{La importancia del contexto y su comprensión}

Este humanismo se construye con base en diversas apreciaciones filosóficas que deben ser consideradas relevantes. En esa línea, el pensamiento de Gilbert Simondon (2009) representa un ineludible soporte del humanismo tecnológico, ya que da prioridad al reconocimiento de los procesos que constituyen la individuación, permitiendo esclarecer en muchos casos las implicaciones que pueden llegar a tener determinadas tecnologías para nuestras vidas, y más aún, teniendo en cuenta el enorme poder que la IA está adquiriendo.

La filosofía simondiana sostiene que el esquema subjetivista, que separa radicalmente sujeto de objeto, subjetividad de objetividad, podría ser cuestionado a la luz de los objetos técnicos, pues la historia demuestra que el sujeto pensante no supone un punto de partida sólido, ya que su intencionalidad se encuentra también determinada por aquellos objetos técnicos a los que supuestamente pretende determinar. Se trataría de un proceso relacional donde sujeto y objeto se determinan mutuamente. Escribe Simondon (2009):

El método consiste en no intentar componer la esencia de una realidad mediante una relación conceptual entre dos términos extremos, y en considerar toda verdadera relación como teniendo rango de ser. La relación es una modalidad del ser; es simultánea respecto a los términos cuya existencia asegura. Una relación debe ser captada como relación en el ser, relación del ser, manera del ser y no simple relación entre dos términos a los que podríamos conocer adecuadamente mediante conceptos ya que tendrían una efectiva existencia separada. Es porque los términos son concebidos como sustancias que la relación es relación entre términos, y el ser es separado en términos porque es primitivamente, anteriormente a todo examen de individuación, concebido como sustancia (p. 37).

La identificación de este proceso relacional y la determinación dialéctica de la individuación permite la toma de conciencia de las profundas transformaciones que la tecnología representa para la existencia humana y de los usos que se pueden hacer de esta, ya sea en términos positivos o negativos. 
Los contextos son determinantes y constituyen al humano y a lo no humano. Debemos asumir la idea que afirma que el sujeto no determina enteramente al objeto, que en este caso sería el artefacto, pues ahí también se encuentra presente un medio que es determinante. La constitución ontológica de la tecnología no responde únicamente a la intencionalidad del creador, ya que está insertada dentro de un medio que lo determina. Para vislumbrar las fuerzas e intencionalidades que se encuentran tras la IA, es necesario reconocer que son diversos los factores que determinan y condicionan la constitución, más allá de la simple relación sujeto y objeto, subjetivo y objetivo, hay más aspectos a tener en cuenta, pues, como se ha señalado, la individuación es fruto de un proceso relacional. La individualización representa el acto de reconocer que existe un medio asociado, a la vez natural y técnico, que incide, condiciona y determina la constitución de los objetos técnicos, y que a la vez provoca una interacción con los sujetos. El ser técnico se autocondiciona y a la vez condiciona a los sujetos, generando un conjunto de posibilidades de interacción entre el mundo y el objeto, entre el objeto y el mundo, el hombre y el objeto, el objeto y el hombre, el hombre y el mundo, etc., que a fin de cuentas refieren a la pluralidad de factores que emergen en la constitución del ser técnico. Lo dicho hasta ahora significa que el ser de la técnica no es impuesto exclusivamente por el ser humano, como si de una esencia estática se tratara, sino que el devenir va marcando su concretización con base en una diversidad de factores. Reconocer este hecho supone darse cuenta de que la tecnología es un medio de aprovechamiento para el florecimiento del ser humano que es consciente de sus límites.

La comprensión de la técnica no puede desligarse del mundo humano, pues es una expresión de la relación del hombre con la realidad. Cuando el hombre crea objetos técnicos, ejerce una función inventiva que supone una anticipación y un acercamiento a la vida. La creación de objetos técnicos es una invención de vida, una obra de vida, una incidencia sobre la realidad en la creación de nuevas formas que cuentan con finalidades que son pensadas pero que también se van constituyendo en el devenir plural.

\section{Humanismo tecnológico y nuevo modo de obrar humano}

La forma de obrar del ser humano requiere de una transformación ante las exigencias éticas que demanda el nuevo tiempo tecnológico. El análisis de Jonas (1995, p. 58-59), en lo referente al vacío ético y a la necesidad de nuevos planteamientos axiológicos, es muy oportuno para la formulación del humanismo tecnológico. El humanismo tecnológico no solo se construye sobre la idea de una tecnología al servicio de la existencia del ser humano y su entorno para un favorable florecimiento, sino también sobre un soporte de responsabilidad que reconozca las exigencias de una nueva ética y sobre el que no sería posible hablar posteriormente de una IAR.

El ritmo de crecimiento vertiginoso del campo de la IA confirma el pronóstico de Jonas acerca del salto cualitativo que ha experimentado el poder tecnológico a partir de la alianza entre la técnica y las ciencias naturales. No obstante, el salto no es únicamente cualitativo, sino también cuantitativo, pues ha aumentado su espectro de extensión a diversas dimensiones de la vida humana. En el diseño tecnológico se proyectan determinados sentidos que posteriormente pueden tener un impacto fundamental sobre la vida, de modo que esos sentidos proporcionan una significación que debe ser considerada desde el principio ético de la responsabilidad. Como señalan Sepúlveda Férriz y Moratalla: (2011)

[...] la tecnología exige responsabilidades porque la creación técnica es una forma de dotar de sentido y este puede significar dominación, alienación, barbarie, cierre del horizonte humano. Así, puesto que también Jonas reconoce que no es posible renunciar a la tecnología, resulta necesaria la reflexión sobre la legitimidad de la acción tecnológica (p. 7).

Esta técnica moderna fundamentada en la máxima baconiana de que "saber es poder" depara importantes desafíos para la humanidad y la biosfera que demandan que el humanismo se reformule en base a nuevas exigencias éticas. Aunque el planteamiento jonasiano centre su preocupación en aspectos bioéticos, sería 
importante afirmar que puede ser extensible a otros ámbitos, como el profesional o el militar, ya que al fin y al cabo se promueve la necesidad de reflexionar sobre las implicaciones que tiene la técnica moderna para las generaciones futuras y el bienestar vital. Además, la transformación del obrar humano con la incorporación y contextualización de ciertos principios éticos serviría para plantear la exigencia en la adopción de estrategias de precaución frente a determinadas actuaciones tecnológicas que podrían estar siendo asumidas de modo irreflexivo y carente de crítica.

¿Es necesario un nuevo humanismo para las nuevas exigencias éticas? Podríamos afirmar que sí, principalmente porque el poder que subyace en la tecnologías plantea una transformación de nuestro obrar. El humanismo tecnológico representa una superación de la concepción antropocéntrica propia de la Modernidad y nos sitúa ante un relato crítico frente al nihilismo tecnológico y la actitud acrítica en el ideal de progreso. La transformación del obrar implica una nueva concepción del mundo y la naturaleza donde se incorporen componentes de valoración y bondad, fruto de una exigencia que nace del ser. Esta exigencia de carácter ontológico se encuentra bajo el paraguas de una concepción antropológica en la que la responsabilidad frente al mundo se conciba a modo de una obligación moral, como sostiene Moratalla (2007, p. 373).

Cuando se afirma que el humanismo tecnológico representa una condición de posibilidad para poder plantear una IAR, es porque el ser humano debe reflexionar y reorientar su relación con la tecnología para que ésta incorpore un sentido de responsabilidad con el mundo. Necesariamente eso no sería posible sin plantear la posibilidad de una transformación del obrar humano que surja desde una matriz antropológica, como señala Jonas (1995, pp. 32-38). El humanismo tecnológico se construye sobre la base de un imperativo ético que implica la transformación del obrar, una premisa que resulta ser fundamental para proyectar una IAR.

\section{Conclusión}

Queda un largo camino por recorrer para expandir el espíritu del humanismo tecnológico en un tiempo que nos empuja cada vez a mayor velocidad para dar respuestas a las problemáticas que enfrenta la humanidad. Por ello, es fundamental comenzar a plantear este tipo de humanismo en aquellas áreas en las que se llevan a cabo los estudios, diseños y regulaciones de las tecnologías más avanzadas. En el contexto de este artículo, la tecnología avanzada que ha sido propuesta para orientar la reflexión es la IA.

En torno al campo de la IA surgen importantes desafíos que condicionarán el futuro de la humanidad en las próximas décadas. En ese sentido es muy importante la incorporación de criterios éticos para promover un concepto de IAR que enfrente los desafíos plantee alternativas en provecho y beneficio para la humanidad. Una premisa esencial para promover ese concepto consiste en asumir la necesidad de un humanismo tecnológico en el que se cultiven un conjunto de ideas que proyecten un sentido de compromiso y florecimiento en los sistemas artificiales para el beneficio de la humanidad y su entorno.

Un buen comienzo para impulsar este humanismo tecnológico que contribuya al planteamiento de una IAR, debería consistir en un fortalecimiento de la ética y la filosofía en los campos del saber destinados al estudio de la tecnología. La tecnología no es una creación axiológicamente neutra, como señalaría con acierto en su día Lévy-Leblond (1975), al sostener que la ciencia despliega su actividad en contextos influenciados por intereses de diversa índole. En ese sentido, especialmente los tecnólogos, pero también la ciudadanía en general, tendría que someterse a un proceso de formación ética y filosófica para poner de relieve aquellas problemáticas que giran en torno a la tecnología más avanzada y cuáles son las oportunidades que contribuir al florecimiento y bienestar. El impulso hacia un humanismo tecnológico supone un importante reto para las próximas décadas, y de él depende el espíritu que se proyectará sobre aquellas tecnologías que condicionarán la vida. 


\section{Bibliografía}

Aguirre Romero, J. M. (2002) "Ciencia, Humanismo, Humanidades y Tecnología. Espéculo”, Revista de estudios literarios. Disponible en: http://webs. ucm.es/info/especulo/numero1g/humanism.html [Consulado 04-06-2019)

Carr, N. (2014 Atrapados por las máquinas. Cómo las máquinas se apoderan de nuestras vidas. Madrid: Alfaguara.

Della Mirandola, P. (1984) De la dignidad del hombre. Madrid: Editora Nacional.

Dewey, J. (1948) La experiencia y la naturaleza. México: Fondo de Cultura Económica.

Domingo Moratalla, T. (2007) "La ética antropológica de Hans Jonas en el horizonte de la fenomenología hermenéutica", Thémata: Revista de Filosofía, 39, pp. 373-380.

Ellul, J. (2003) La edad de la técnica. Barcelona: Octaedro

Ellul, J. (2004) “El orden tecnológico”. En: C. Mitcham y R. Mackey. Eds. Filosofía y tecnología. Madrid: Ediciones Encuentro.

Horkheimer, M. (2010) Crítica de la razón instrumental. Madrid: Trotta.

Horkheimer, M. y Adorno, T. (2016) Dialéctica de la Ilustración: fragmentos filosóficos. Madrid: Trotta.

Huxley, A. (2017) Literatura y ciencia. El humanismo frente al progreso científico y tecnológico. Barcelona: Página Indómita.

Jonas, H. (1995) El principio de responsabilidad. Ensayo de una ética para la civilización tecnológica. Barcelona: Herder.
Jonas, H. (1997) Técnica, medicina y ética: sobre la práctica del principio de responsabilidad. Barcelona: Paidós.

Jonas, H. (2001) Más cerca del perverso fin y otros diálogos y ensayos. Madrid: Los libros de la Catarata.

Jonas, H. (2005) Memorias. Oviedo: Losada.

Lévy-Leblond, J. M. (1975) La ideología de/en la física contemporánea y ostros ensayos. Barcelona: Editorial Anagrama.

Molinuevo, J. L. (2004) Humanismo y nuevas tecnologías. Madrid: Alianza Editorial.

Ortega y Gasset, J. (1965) Meditación de la técnica. Madrid: Editorial Espasa-Calpe.

Parra, N.-H. y Arenas-Dolz, F. (2015) Revolución tecnológica y democracia del conocimiento. Por una universidad innovadora. Valencia: Laboratorio de la Sociedad del conocimiento.

Sartre, J. P. (1999) El existencialismo es un humanismo. Barcelona: Edhasa.

Sepúlveda Férriz, J. L. y Domingo Moratalla, T. (2011) “La transformación del obrar humano en la época de la civilización tecnológica y la exigencia de una nueva ética”, Principios: Revista de Filosofía, 18 (30).

Simondon, G. (2009) La individuación a la luz de las nociones de forma y de información. Buenos Aires: Cactus-La Cebra.

Trías, E. (200o) Ética y condición humana. Barcelona: Península.

Winner, L. (2008) La ballena y el reactor. Barcelona: Gedisa. 